\title{
AVALIAÇÃO TERAPEUUTICA DO OLTIPRAZ NA INFECÇÃO HUMANA PELO S. MANSONI
}

\author{
Silvino Alves de CARvalHo (1), Vicente AMATo NETO (2), José Murilo Robilotta zEITUNE (3), \\ Marla Aparecida SHIKANaI-YaSUDA (4) \& Clovis Kiomitsu TAKIGUTI (5)
}

\section{R E S U M O}

Foram tratados com oltipraz, dose oral única de $30 \mathrm{mg} / \mathrm{kg}$ de peso, 72 indivfđuos com esquistossomose mansoni, matriculados na Clínica de Doenças Infecciosas e Parasitárias do Hospital das Clínicas da Faculdade de Medicina da Universidade de São Paulo. As idades dos pacientes variaram de 7 a 58 anos, sendo que $8(11,1 \%)$ eram menores de 15 anos. Os principais efeitos colaterais consistiram em tonturas $22,2 \%$, sonolência $22,2 \%$, náuseas $22,2 \%$, cefaléia, $9,7 \%$, astenia $9,7 \%$, parestesia $8,3 \%$, vômitos $8,3 \%$, cólicas $7,0 \%$, diarréia $4,2 \%$, escotomas $2,8 \%$, sialosquese $2,8 \%$, nódulos dolorosos em extremidades $2,8 \%$ e outras manifestações clínicas em menor frequiência. A toxicidade do medicamento foi avaliada mediante a realização pré e pos-tratamento, de exames hematimétricos, de função renal (uréia e creatinina), hepática (enzimas de liberação hepato-canalicular e bilirrubinas), cardíaca (ECG) e neuropsiquiátrica (EEG). Não foram encontrados nos controles laboratoriais alteraçóes relevantes ou que determinasse alguma repercussão clínica. O controle de cura verificou-se em 49 indivíduos, através de 8 coproscopias (no período de 6 meses subsequiente ao tratamento) utilizando-se duas técnicas (Hoffman e Kato/Katz) para cada amostra de fezes. Dos 29 indivíduos que tiveram as 8 coproscopias negativas, 20 realizaram biopsia retal, mostrando-se positiva em uma oportunidade, indicando $5 \%$ de "falsos negativos" com relação às coproscopias. $O$ indice de cura para todas as faixas etárias foi de $59,2 \%$. Os resultados obtidos demonstraram ser o oltipraz de relativa eficácia e determinante de efeitos tóxicos-colaterais sistêmicos no tratamento da esquistossomose mansoni.

UNITERMOS: Esquistosomose mansonica humana - Terapia pelo oltipraz

\section{N T RO D U G A O}

OItipraz (4-metil-5-(2-pirazinil)-3H-1,2 ditio- - co de açăo esquistossomicida, sintetizado em le-3 tione) se constitui em um composto quimi- 1975 pela "Rhone-Poulenc Research Center". Sua

(1) Professor-assistente da Clínica de Doenças Infecciosas e Parasitárias da Faculdade de Medicina da Universidade de Săo Paulo

(2) Professor-titular da Clínica de Doenças Infecclosas e Parasitárias da Faculdade de Medicina da Universidade de São Paulo

(3) Médico-assistentee da Disciplina de Gastroenterologia do Departamento de Clínica Médica do Hospital das Clínicas da Faculdade de Medicina da Universidade de Săo Paulo

(4) Professora-assistente doutora da Clínica de Doenças Infecciosas e Parasitárias da Faculdade de Medictna da Univer. sidade de São Paulo

(5) Professor-doçente livre do Departamento de Medicina Preventiva da Faculdade de Medicina da Universidade e Säo Paulo Endereço: Clínica de Doenças Infecciosas e Parasitérias da Fraculdade de Medicina da USP. Rua Dr. Eneas de Carvalho Aguiar, 255. CEP 05403 - Săo Paulo, SP - Brasil 
CARVALHO, S. A. de; AMATO NETO, V.; ZeITUNE, J. M. R.; SHIKANAI-YASUDA, M. A. \& TAKIGUT, C. K. Avaliaçăo terapéutica do oltiprað na infecçăo humana pelo s. mansoni. Rev. Inst. Med. trop. Säo Paulo, 28: 271-277, 1986 .

absorção é gastrointestinal e sua eliminação se dá pela urina e fezes através de conjugados metabólitos 8 .

Ensaios clínico-terapêticos demonstraram ter o oltipraz ação farmacodinâmica ativa às várias espécies de esquistossomose do homem, bem como desprovido de efeitos tóxico-colaterais de maior relevânicia $1,4,5,6,7,8$.

Nosso objetivo no presente estudo consistiu em avaliar a eficácia e toxicidade do oltipraz em individuos infectados com o $\mathbf{S}$. mansoni.

\section{MATERIAL E MÉTODOS}

Constituiu-se de 72 indivíduos infectados com o S. mansoni, de ambos os sexos, com idade variável de 7 a 58 anos, matriculados na Clínica de Doenças Infecciosas e Parasitárias do Hospital das Clínicas da Universidade de São Paulo. O diagnóstico de parasitose, na totalidade dos casos, deu-se através da coproscopia (qualitativa e quantitativa).

A maioria dos pacientes procediam do Nordeste (BA, PE, AL, SE) e Leste (MG) do país. Dos 72 pacientes tratados, $(11,1 \%)$ eram menores de 15 anos (Tabela I). A grande maio-

T A B E I A. I

Distribuiçăo do n. $\mathrm{e} \%$ dos pacientes tratados com oltipraz segundo as faixas etárias

\begin{tabular}{lrr}
\hline Faixa etária & (N.0) & $(\%)$ \\
\hline $5-15$ & $B$ & 11,1 \\
$16-25$ & 24 & 33,3 \\
$26-35$ & 30 & 41,7 \\
$36-45$ & 7 & 9,7 \\
46 e mais & 3 & 4,2 \\
\hline Total & 72 & 100,0 \\
\hline
\end{tabular}

ria era assintomáticos ou oligossintomáticos (Tabela II) e a associaçăo mórbida mais freqüente foi a hipertensăo arterial $(9,7 \%)$, seguida de outras em menor frequiência (Tabela III).

As formas clínicoevolutivas predominantes foram a intestinal e hépato intestinal (Tabela IV). A coproscopia quantitativa mostrou serem leves a grande maioria das infecções (Tabela v).
T A E I A II

Distribufçăo do n. $\mathrm{e} \%$ dos paicentes tratados com oltipraz, segundo os sintomas referidos antes do tratamento

\begin{tabular}{|c|c|c|}
\hline Sintomas $(*)$ & $\left(\mathrm{N} .0^{\circ}\right)$ & $(\%)$ \\
\hline Sem queixas & 26 & 36,0 \\
\hline Cólicas abdominais & 24 & 33,3 \\
\hline Obstipaçăo intestinal & 11 & 15,3 \\
\hline Náuseas & 7 & 9,7 \\
\hline Diarréia & 6 & $B, 3$ \\
\hline Cefaléia & $\mathbf{5}$ & 7,0 \\
\hline Tonturas & $\mathbf{5}$ & 7,0 \\
\hline Dispnéia & $\mathbf{5}$ & 7,0 \\
\hline Emagrecimento & 5 & 7,0 \\
\hline Anorexia & 5 & 7,0 \\
\hline Palpitaçōes & 3 & 4,2 \\
\hline Empachamento & 3 & 4,2 \\
\hline Disenteria & 2 & 2,8 \\
\hline Pirose & $\mathbf{2}$ & 2,8 \\
\hline Dores articulares & 2 & 2,8 \\
\hline Dor torácica & 1 & 1,4 \\
\hline Convulsão/Desmaio & 1 & 1,4 \\
\hline Epistaxe & 1 & 1,4 \\
\hline Disturbio de comportamento & 1 & 1,4 \\
\hline Edema dos membros inferiores & 1 & 1,4 \\
\hline
\end{tabular}

(*) Alguns pacientes apresentaram mais de um sintoma

T A B E L A III

Distribufçäo do n.० e \% dos pacientes tratados com oltipraz. segundo doenças intercorrentes

\begin{tabular}{|c|c|c|}
\hline Doenças intercorrentes (*) & $(N .0)$ & $(\%)$ \\
\hline Sem doenças diagnosticadas & 34 & 47,0 \\
\hline Hipertensão arterial & 7 & 9,7 \\
\hline Inteoção e/ou Doença de Chagas & $\mathbf{5}$ & 6,9 \\
\hline Disritmia cerebral & 3 & 4,2 \\
\hline Hipodesenvolvimento pondero/estatural & 3 & 4,2 \\
\hline Bronquite asmática & 2 & 2,8 \\
\hline Hérnia inguinal & 2 & 2,8 \\
\hline Hérnla opigástrica & 1 & 1,4 \\
\hline Gastrite & 1 & 1,4 \\
\hline Bócio multinodular & 1 & 1,4 \\
\hline Hipotireoidismo & 1 & 1,4 \\
\hline Neurite do ciático & 1 & 1,4 \\
\hline Neurite optica & 1 & 1,4 \\
\hline Carcinoma do colo uterino & 1 & 1,4 \\
\hline Mfoma uterino & 1 & 1,4 \\
\hline Artrite reumatoide & 1 & 1,4 \\
\hline Espondilite anquilosante & 1 & 1,4 \\
\hline Uretrite nâto gonococica & 1 & 1,4 \\
\hline Psicose pós-parto & 1 & 1,4 \\
\hline Rinite alérgica & 1 & 1,4 \\
\hline Cisto do tireoglosso & 1 & 1,4 \\
\hline Esquizofrenia & 1 & 1,4 \\
\hline Esclerose muiltipla & 1 & 1,4 \\
\hline Total & 72 & 100,0 \\
\hline
\end{tabular}

(*) Doenças intercorrentes diagnosticadas que motivaram ou não a consulta.

Os pacientes antes da terapêtica com o ol tipraz eram submetidos a exames clínico e la. boratoriais, visando avaliar as funções cardíaca, renal, hepática, hematológica e neuropsiquiá 
Carvalho, S. A. de; aMato Neto, v.; Zeitune, J. M. R.; ShIKanaI.Yasuda, M. A. \& TaKiguTI, C. K. Avaliaçăo terapéutica do oltipraz na infecçāo humana pelo S. mansoni. Rev, Inst. Med. trop. Säo Paulo, 23; 271-277, 1986.

T A B E L A IV

Distribuiçăo do n.० e \% dos prcientes tratados com oltipraz, segundo as formas clínicas

\begin{tabular}{lcr}
\hline Formas clínicas & (N.०) & $(\%)$ \\
\hline Intestinal & 41 & 57,0 \\
Hépato-intestinal & 25 & 34,7 \\
Hépato-esplénica & 6 & 8,3 \\
\hline Total & 72 & 100,0
\end{tabular}

T A B E L A V

Distribuição do n.॰ e \% dos pacientes tratados com oltipraz, segundo a "carga parasttária" (n..$^{\circ}$ de ovos/g de fezes)

\begin{tabular}{lrr}
\hline N.0 de ovos $/ g$ de fezes & $(N .0)$ & $(\%)$ \\
\hline $96-500$ & 53 & 73,6 \\
$501-1000$ & 11 & 15,3 \\
$1001-1500$ & 5 & 6,9 \\
1501 ou mals & 3 & 4,2 \\
\hline Total & 72 & 100,0 \\
\hline
\end{tabular}

trica e que serviriam como parâmetros de avaliação de toxicidade do medicamento após o tratamento.

O altipraz foi empregado na dosagem de $30 \mathrm{mg} / \mathrm{kg}$ de peso para todas as faixas etárias. Após a administração do medicamento os pa. cientes eram colocados em repouso relativo por 4 horas, zempre sob supervisão do serviço de enfermagem, orientado no sentido de anotar qualquer efeito colateral que eventualmente pudesse surgir no periodo de observação. Quando do primeiro retorno subsequiente ao tratamento, o paciente era submetido a anamnese e exame físico, no sentido de se detectar possiveis manifestaçóes colaterais de aparecimento mais tardio.

Os controles bioquímicos (enzimas hepáticas, bilirrubinas, coagulograma, eletroforese de proteínas, uréia, creatinina e glicemia), hematimétricos e eletrocardiograficos foram realizados antes e no $7 .^{\circ}$ e $14 .^{\circ}$ dia após o tratamento. A eletroencefalografia foi realizada em 20 individuos antes e mais ou menos 2 horas apos a administração do medicamento.

O controle de cura baseou-se em 8 coproscopias utilizando-se duas técnicas (Hoffman e Kato/Katz) para cada amostra de fezes. Quatro coproscopias foram realizadas com intervalos mensais e quatro com intervalos quinzenais. Se negativas as coproscopias o paciente era submetida a biopsia retal.
Nos controles bioquímicos e hematimétri $\cos$ os testes da igualdade de média foram efe tuados utilizando-se a estatística " $t$ ", supondose igualdade de variância. Adotou-se um nivel de significância igual a 0,05 .

\section{RESULTADOS}

O oltipraz de um modo geral não foi bem tolerado, haja vista que $63,9 \%$ dos indivíduos tratados apresentaram um ou mais efeito colateral (Tabela .VI). As reaçóes gastrointesti-

T A $\mathbf{E}$ I A VI

Distribuiçāo do n.० e \% dos pacientes tratados com oltipraz, que apresentaram ou năo manifestações colaterals.

\begin{tabular}{|c|c|c|c|c|c|}
\hline \multicolumn{2}{|c|}{ Manifestaçōes colaterais $\left({ }^{\star}\right)$} & \multirow[t]{2}{*}{. } & \multirow{2}{*}{$\frac{(N .0)}{26}$} & & \multirow{2}{*}{$\frac{(\%)}{36,1}$} \\
\hline Sem queixas & & & & & \\
\hline Tonturas & $\cdot$ & & 16 & & 22,2 \\
\hline Sonoléncia & & & 16 & & 22,2 \\
\hline Náuseas & & & 16 & & 22,2 \\
\hline Cefaléis & & & 7 & & 9,7 \\
\hline Astenis & & & 7 & & 9,7 \\
\hline Vômitos & $\therefore$ & & 6 & & 8,3 \\
\hline $\begin{array}{l}\text { Parestesias (dolorosas } \\
\text { a) membros superiores } \\
\text { b) lábio e língua (1) } \\
\text { c) generalizada (1) }\end{array}$ & $\begin{array}{l}\text { ou não) } \\
\text { s e inferiores }\end{array}$ & (4) & 6 & . & 8,3 \\
\hline Dor abdominal & & & $\mathbf{5}$ & & 7,0 \\
\hline Diarréia & & & 3 & & 4,2 \\
\hline $\begin{array}{l}\text { Erupção cutânea } \\
\text { a) exantema ( } 2 \text { ) } \\
\text { b) urticária generaliza }\end{array}$ & (1) & & 3 & . & 4,2 \\
\hline Escotomas & & & 2 & & 2,8 \\
\hline Sialosquese & & & 2 & & 2,8 \\
\hline Nodulos dolorosos nas & extremidades & & $\mathbf{2}$ & & 3,8 \\
\hline Eritema nodoso & & & 1 & & 1,4 \\
\hline Lacrimejamento & & & 1 & & 1,4 \\
\hline Soluços & $\cdot$ & & 1 & & 1,4 \\
\hline Colúria & & & 1 & & 1,4 \\
\hline
\end{tabular}

nais surgiram na grande maioria dos casos nas três primeiras horas após a medicação, ao contrário de outros efeitos tóxico-colaterais de caráter sistêmico (astenia, parestesias, erupçōes cutâneas, nódulos dolorosos de extremidades, eritema nodoso e escotomas) que surgiram durante os sete primeiros dias que se seguiram ac tratamento.

Dos 72 individuos tratados, 40 tinham o eletrocardiograma sem alterações antes do tra. tamento e 8 apresentaram uma ou mais alterações no traçado nos controles pós-tratamento: arritmias sinusal (3), alteração de repolarização 
CARVALHO, S. A. de; AMATO NETO, V.; ZEITUNE, J. M. R.; SHIKANAI-YASUDA, M. A. \& TAKIGUTI, C. K. Avaliação terapêutica do oltipraz na infecção humana pelo $S$. mansonil. Rev. Inst. Med. trop. São Paulo, 28: 271-277, 1986.

ventricular (3), extrasistolia (2), bloqueio A-V do $10^{\circ}$ grau (1) e sobrecarga ventricular esquerda (1).

A eletroencefalografia realizada em 20 pacientes não evidenciou em nenhum inđivíduo qualquer alteração no traçado.
Nos controles hematimétricos houve uma diminuição dos monócitos (2. ${ }^{2}$ semana) e elevação dos linfócitos (1.a semana) estatisticamente significantes. Na bioquímica do sangue de significância estatística ocorreu diminuição da bilirrubina indreta na $2 .^{a}$ semana (Tabela VII e VIII)

T A B I I A VII

Médias, desvios pađrão e estatística " $t$ " das variáveis estudadas nas fases pré-tratamento e 7 dias pós-tratamento em pacientes esquístossomóticos tratados com oltipraz

\begin{tabular}{|c|c|c|c|c|}
\hline \multirow{2}{*}{ Variável } & \multicolumn{2}{|c|}{ Média \pm desvio padrão } & \multirow{2}{*}{$\begin{array}{l}\text { Número de } \\
\text { pacientes }\end{array}$} & \multirow{2}{*}{$\begin{array}{l}\text { Estatistica } \\
\text { " } t " \text { " }\end{array}$} \\
\hline & Pré-tratamento & 7 dias & & \\
\hline Hemácias $\times 10^{8}$ & $4,9 \pm 0,6$ & $4,9 \pm 0,6$ & 15 & 0,00 \\
\hline Hematócrito & $43,9 \pm 4,2$ & $44,2 \pm 4,0$ & 13 & 0,79 \\
\hline Hemoglobina & $13,5 \pm 1,6$ & $13,6 \pm 1,7$ & 31 & 1,26 \\
\hline Leucocitos $\times 1000$ & $7,1 \pm 1,9$ & $7,5 \pm 2,5$ & 32 & 0,86 \\
\hline Neutrófilo segmentado & $54,1 \neq 8,8$ & $49,2 \pm 12,9$ & 18 & 1,75 \\
\hline Neutrófilo bastonetes & $3,9 \pm 2,7$ & $3,3 \pm 2,3$ & 18 & 1,05 \\
\hline Linfócitos & $29,3 \pm 9,0$ & $34,0 \pm 9,4$ & 18 & $2,27\left(^{\circ}\right)$ \\
\hline Monócitos & $4,5 \pm 3,6$ & $4,0 \pm 2,3$ & 18 & 0,55 \\
\hline Eosinófilos & $6,5 \pm 5,2$ & $9,3 \pm 8,3$ & 18 & 1,89 \\
\hline Fosfatase alcalina & $158,7 \pm 48,8$ & $162,3 \pm 59,1$ & 31 & 0,58 \\
\hline Aspartato amino transferase (TGO) & $13,0 \pm 4,5$ & $12,4 \pm 3,4$ & 27 & 0,98 \\
\hline Alanina amino transferase (TGP) & $14,5 \pm 6,4$ & $13,7 \pm 6,1$ & 32 & 0,76 \\
\hline Gama glutamil transferase (GT) & $20,8 \pm 15,2$ & $20,0 \pm 15,7$ & 31 & 0,57 \\
\hline Dehidrogenase láctica (DFL) & $174,2 \pm 35,6$ & $181,1 \pm 41,4$ & 32 & 0,93 \\
\hline Leucina amino asilamidase (LAP) & $16,2 \pm 4,7$ & $16,2 \pm 3,7$ & 32 & 0,05 \\
\hline Bilirrubina direta & $0,3 \pm 0,1$ & $0,2 \pm 0,1$ & 31 & 0,86 \\
\hline Bilirrubina indireta & $0,3 \pm 0,2$ & $0,3 \pm 0,1$ & 31 & 0,97 \\
\hline Proteinas totais & $7,6 \pm 0,6$ & $7,5 \pm 0,6$ & 30 & 0,57 \\
\hline Albumina & $4,3 \pm 0,5$ & $4,2 \pm 0,6$ & 30 & 0,67 \\
\hline Globulina & $3,3 \pm 0,6$ & $3,2 \pm 0,6$ & 30 & 0,62 \\
\hline Alfa-1-globulina & $0,2 \pm 0,1$ & $0,2 \pm 0,1$ & 29 & 0,05 \\
\hline Alfa-2-globulina & $0,6 \pm 0,2$ & $0,6 \pm 0,1$ & 29 & 0,18 \\
\hline Beta-globulina & $0,9 \pm 0,2$ & $0,9 \pm 0,2$ & 30 & 0,98 \\
\hline Gama-globulina & $1,5 \pm 0,4$ & $1,5 \pm 0,5$ & 30 & 0,44 \\
\hline Uréla & $24,6 \pm 8,0$ & $25,0 \pm 0,2$ & 31 & 0,32 \\
\hline Creatinina & $0,9 \pm 0,3$ & $0,9^{\circ} \pm 0,5$ & 31 & 0,75 \\
\hline Glicemia & $85,4 \pm 11,7$ & $89,7 \pm 13,7$ & 18 & 1,81 \\
\hline Tempo de protrombina (TP) & $11,9 \pm 1,2$ & $12,3 \pm 0,8$ & 11 & 0,81 \\
\hline Tempo de trombina (TT) & $14,3 \pm 1,6$ & $14,5 \pm 2,0$ & 11 & 0,31 \\
\hline Ternpo de tromboplastina parcial ativada (TTPA) & $4 B, 0 \pm 6,4$ & $47,1 \pm 1,9$ & 11 & 0,49 \\
\hline
\end{tabular}

(*) Significante ao nfvel de $5 \%$

Dos 72 indivíduos tratados 49 completaram o controle de cura previamente estabelecido. Destes, 20 apresentaram falhas no tratamento, traduzidas pela evidenciação de ovos viáveis nas fezes (19) e na biopsia retal (1). Dos 29 pacientes tratados e que apresentaram as 8 coproscopias negativas, 20 realizaram biópsia da mucosa retal, sendo 1 positivo para ovos viá. veos de S. mansoni, determinando um índice de $5 \%$ de "falsos negativos" com relação às coproscopias. A eficácia do oltipraz para todas as faixas etárias foi de $59,2 \%$. Nos casos em que não se obteve a cura parasitológica, a re cução do número de ovos/g. de fezes foi da ordem de $83,6 \%$ (Tabela IX), 
CARVAlHo, S. A. de; AMATO NETO, V.; zeITUNE, J. M. R.; ShIKANAI.YASUDA, M. A. \& TAKIGUTI, C. K. Avaliaçăo terapêutica do oltipraz na infeç̧ăo humana pelo $S$. mansoni. Rev. Inst. Med. trop. São Paulo, 28: 271-277, 1986.

T A B E L A VIII

Médias, desøios pađrão e estatistica "t" das variáveis estudadas nas fases pré-tratamento e 14 dias pós-tratamento: em pacientes esquistossomóticos tratados com oltipraz

\begin{tabular}{|c|c|c|c|c|}
\hline \multirow{2}{*}{ Variavel } & \multicolumn{2}{|c|}{ Média \pm desvio padrăo } & \multirow{2}{*}{$\begin{array}{l}\text { Numero de } \\
\text { pacientes }\end{array}$} & \multirow{2}{*}{$\begin{array}{c}\text { Estatístict } \\
\text { "t" }\end{array}$} \\
\hline & Pré-tratamento & 14 dias & & \\
\hline Hemácias $\times 10^{\circ}$ & $4,8 \pm 0,4$ & $4,8 \pm 0,4$ & 9 & 0,20 \\
\hline Hemat6crito & $39,9 \pm 4,9$ & $39,6 \pm 2,9$ & 9 & 0,17 \\
\hline Hemoglobins & $13,0 \pm 1,9$ & $13,1 \pm 1,3$ & 14 & 0,11 \\
\hline Leucócitos $\times 1000$ & $7,2 \pm 2,1$ & $7,0 \pm 2,4$ & 14 & 0,48 \\
\hline Neutrófilo segmentados & $55,2 \pm 9,8$ & $50,8 \pm 8,0$ & 11 & 2,00 \\
\hline Neutrofilo bastonetes & $3,9 \pm 3,0$ & $3,6 \pm 2,6$ & 11 & 0,31 \\
\hline Linf 6 citos & $26,9 \pm 6,6$ & $30,7 \pm 9,1$ & 11 & 1,12 \\
\hline Monócitos & $4,9 \pm 3,3$ & $2,7 \pm 1,8$ & 11 & $3,01\left(^{\circ}\right)$ \\
\hline Eosinofilos & $6,4 \neq 5,4$ & $6,9 \pm 8,1$ & 11 & 1,43 \\
\hline Fosfatase alcalina & $199,8 \pm 143,2$ & $211,6 \pm 145,5$ & 13 & 0,93 \\
\hline Aspartato amino transferase (TCO) & $11,6 \pm 3,3$ & $11,5 \pm 2,3$ & 12 & 0,09 \\
\hline Alanina amino transferase (TGP) & $12,1 \pm 5,8$ & $12,0 \pm 5,3$ & 14 & 0,08 \\
\hline Gama glutamil transferase (GT) & $17,6 \pm 19,3$ & $16,7 \pm 13,1$ & 12 & 0,21 \\
\hline Dehidrogenase láctica (DFI) & $187,5 \pm 40,4$ & $18 B, 9 \pm 43,5$ & 8 & 0,08 \\
\hline Leucina amino arilamidase (LAP) & $16,5 \pm 5,0$ & $17,2 \pm 5,6$ & 13 & 0,48 \\
\hline Bilirrubina direta & $0,2 \pm 0,1$ & $0,3 \pm 0,1$ & 12 & 1,16 \\
\hline Bilirrubina Indireta & $0,3 \pm 0,1$ & $0,2 \pm 0,1$ & 12 & $2,59(*)$ \\
\hline Proteinas totals & $7,5 \pm 0,8$ & $7,2 \pm 0,7$ & 14 & 1,18 \\
\hline Albumina & $4,3 \pm 0,6$ & $4,0 \pm 0,5$ & 14 & 1,20 \\
\hline Globulina & $3,2 \pm 0,5$ & $2,9 \pm 0,6$ & 14 & 1,31 \\
\hline Alfa-1-globulina & $0,2 \pm 0,1$ & $0,2 \pm 0,1$ & 14 & 0,56 \\
\hline Alfa-2-globulina & $0,7 \pm 0,1$ & $0,6 \pm 0,2$ & 14 & 1,79 \\
\hline Beta-globulina & $1,0 \pm 0,2$ & $0,9 \pm 0,2$ & 14 & 0,86 \\
\hline Gama-globulina & $1,4 \pm 0,2$ & $1,3 \pm 0,4$ & 14 & 0,74 \\
\hline Uréta & $23,1 \pm 4,4$ & $21,3 \pm 5,3$ & 12 & 1,05 \\
\hline Creatinina & $0,9 \pm 0,2$ & $0,9 \pm 0,2$ & 12 & 0,19 \\
\hline Glicemia & $85,8 \pm 9,1$ & $76,9 \pm 5,4$ & 8 & 1,92 \\
\hline Tempo de protrombina (TP) & $12,7 \pm 1,2$ & $12,2 \pm 0,4$ & 6 & 1,16 \\
\hline Tempo de trombina (TT) & $13,7 \pm 0,8$ & $14,0 \pm 2,3$ & 6 & 0,50 \\
\hline Tempo de tromboplastina parcial ativada (TTPA) & $48,7 \pm 3,4$ & $52,7 \pm 12,2$ & 6 & 0,92 \\
\hline
\end{tabular}

(*) Significante ao nivel de $5 \%$

\section{DISCUSSAO}

No presente estudo o oltipraz demonstrou ser de eficácia relativa e determinante de efei. tos tóxico-colaterais de caráter sistêmico na te. rapêtutica da infecção humana pelo S. mansoni.

Os efeitos colaterais gastrointestinais (co. licas, náuseas, vômitos e điarréia) e neuropsiquiátricos (cefaléia, tonturas e sonolência) fo. ram leves ou moderados. estando em concor. dância com os dados de literatura $1,4,6,7,8$. Contudo, em nossa casuística foram detectados efeitos tóxico-colaterais de caráter sistemico (astenia, parestesias, nódulos dolorosos de extremidades, exupções cutâneas, escotomas, eritema nodoso) que requer estudos complementares para esclarecimentos de sua fisiopatogenia. GENTILINI \& col. ${ }^{4}$, REY \& col. 7 e KATZ \& col. ${ }^{\circ}$ descreveram efeitos tóxico-colaterais com o uso do oltipraz caracterizado por: pareste. sias, dores e contratura das extremidades, com patíveis com alguns dos nossos achados En tretanto o aparecimento de nódulos dolorosos inflamatórios das extremidades, eritema nodo. so e escotomas não tinham ainda registro na literatura sobre o oltipraz.

Em nossa avaliação bioquímica, hematimétrica e eletroencefalográfica não detectamos nenhuma alteração de maior importância e que repercutisse clinicamente, estando em concor: dância com os trabalhos de literatura correlata que revimos $1,4,5,6,7,8$.

Embora tenhamos detectado alteraçōes no traçado eletrocardiográfico após o tratamento, estas foram de caráter transitório e sem reper: cussão clínica.

Quanto à eficácia os dados da literatura nos oferecem indices de cura muito superiores aos 
CARValHo, S. A. de; AMATO NETO, V.; ZeITUNE, J. M. R.; SHIKANAI-YASUdA, M. A. \& TAKIGUTI, C. K. Avaliação terapêutica do oltipraz na infeç̧ão humana pelo S. mansoni. Rev. Inst. Med. trop. Săo Paulo, 28; 271-277, 1986.

T A B I A IX

Distribuição dos pacientes tratados com oltipraz, que apresentaram falha no tratamento, segundo a idade, forma clínica e "carga parasitária" antes e pós o tratamento

\begin{tabular}{|c|c|c|c|c|}
\hline \multirow{2}{*}{ N.o } & \multirow{2}{*}{ Idade } & \multirow{2}{*}{$\begin{array}{c}\text { Forma } \\
\text { clínica (*) }\end{array}$} & \multicolumn{2}{|c|}{ Ovos/g de fezes } \\
\hline & & & Pré & Pós-tratamento \\
\hline 1 & 30 & HE & 168 & 144 \\
\hline 2 & 25 & HI & 240 & 48 \\
\hline 3 & 42 & HI & 384 & 72 \\
\hline 4 & 35 & $I$ & 1248 & 24 \\
\hline 5 & 29 & HI & 744 & 48 \\
\hline 6 & 37 & I & 1080 & 48 \\
\hline$\eta$ & 32 & $I$ & 552 & 120 \\
\hline B & 35 & I & 408 & 48 \\
\hline 9 & 30 & I & 192 & 24 \\
\hline 10 & 26 & HII & 360 & 48 \\
\hline 11 & 33 & I & 120 & 96 \\
\hline 12 & 31 & $\bar{I}$ & 264 & 72 \\
\hline 13 & 12 & I & 240 & 24 \\
\hline 14 & 55 & $\mathbf{I}$ & 144 & 48 \\
\hline 15 & 29 & $\mathbf{I}$ & 96 & 0-Biópsia retal \\
\hline 16 & 27 & $\mathbf{I}$ & 144 & 24 \\
\hline 17 & 24 & HI & 840 & 360 \\
\hline 18 & 33 & I & 288 & 72 \\
\hline 19 & 20 & HI & 648 & 48 \\
\hline 20 & 27 & $I$ & 480 & 48 \\
\hline
\end{tabular}

(*) $\mathrm{I}=$ Intestinal, $\mathrm{HI}=$ Hépato-intestinal e HE $=$ Hépatoesplénica.

nossos ou seja de $59,2 \%$. BELLA \& col. 1 em. pregando dosagem de 25 e $35 \mathrm{mg} / \mathrm{Kg}$ de peso, obteve índice médio de cerca de $94 \%$. GENTI. LINI \& col. ${ }^{4}$, utilizando dose total de 3 a $5 \mathrm{~g}$ durante 2 a 5 dias obteve $100 \%$ de cura. KATZ \& col.5,6 empregando a mesma dosagem por nós utilizada (ou seja $30 \mathrm{mg} / \mathrm{kg}$ de peso dose única) encontrou em duas oportunidades indices de 100,0 e $81,2 \%$ de cura parasitológica.

Concluímos que o oltipraz apresenta eficácia relativa contra o S. mansoni em infecção humana $(59,2 \%$ de cura parasitológica) inferior aos índices por nós detectados no emprego do oxamniquine $\left(82,8 \%\right.$ ) e o praziquantel $(71,3 \%)^{2,3}$. Por outro lado o oltipraz determinou efeitos tóxico colaterais de carâter sistêmico, que requerem estudos ulteriores para efetivos esclarecimentos.

\section{SUMMARY}

Therapeutical investigation of oltipraz in human infection due to $\mathrm{S}$. mansoni

Seventy two patients with schistosomiasis mansoni, registered in the Infectious and $\mathrm{Pa}$ - rasitic Disease Ward, Hospital das Clínicas, Fa. culty of Medicine, University of São Paulo, were treated with a single oral dose of oltipraz, $30 \mathrm{mg} / \mathrm{kg}$ body wcight. Their ages ranged from seven to 58 years and eight patients (11.1\%) were under fifteen. The main side effects found in this trial were: dizziness $22.2 \%$; urowsiness $21 \%$; nausea $22.2 \%$; headache $9.7 \%$; asthenia $9,7 \%$; paresthesia $8.3 \%$; vomiting $3.3 \%$; abdominal cramps $7.0 \%$; diarrhea $4.2 \%$; dry mouth $2.8 \%$; tender nodules in extremities 2.8\%; exanthema $2.8 \%$. Other complaints were seen less often. The drug toxicity was evaluat. ed by workout - blood counts, kidney function tests (BUN and creatinine), hepatic function iest (serum enzymes and bilirubin), EKG and EEG - performed before as well as after treat ment. No relevant abnormality. was found. Success of therapy was assessed in 49 patients through eight consecutive stool examinations, performed during the six-month period follow. ing the drug administration. Both Hoffman's and Kato-Katz's techniques were used. Rectal biopsies were carried out in 20 patients out of 29 whose stool examinations were negative and just one yielded positive, indicating a $5 \%$ rate of false negative examinations. The overall cure raio was $59.2 \%$. The present results point out that oltipraz at the used dose levels is relatively efficaceous in the treatment of schistosomiasis mansoni. Its toxic reactions and side effects on the other hand require further investigation.

\section{AGRADECIMENTOS}

Ao Dr. Aluisio Augusto Cotrim Segurado pela ajuda na realização do presente trabalho.

\section{REFERENCIAS BIBLIOGRAFICAS}

1. BELLA, H.; RAHIM, A. G. A.; MUSTAFA, M. D.; AFMED, M. A. M.; WASFI, S. \& BENETT, J. L. - Oltipraz Antischistosomal efficacy in Sudanese infected with Schistosoma mansonl. Amer. J. trop. Med. Hyg., 31: $775-778$, 1982.

2. CARVALHO, S. A. - Aspectos epidemiológicos e qui. mioterápicos na esquistossomose mansoni. Contribuiçăo ao estudo da quimioterapia pelo oxamniquine. São Paulo, 1978. (Dissertaçāo de mestrado - Faculdade de Medicina da USP).

3. CARVALHO, S. A.; AMATO NETO, V.; ZEITUNE, J M. R.; GOLDBAUM, M.; CASTILHO, E. A. \& GROSSMAN, 


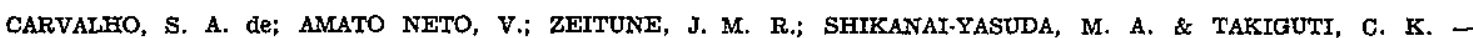
Avaliação terapêtica do oltipraz na infeç̧ăo humana pelo S. mansont. Rev. Inst. Med. trop. São Paulo, 28: $271-277,1986$.

R. M. - Avaliação terapêutica do praziquantel (Embay 8440) na infecção humana pelo $S$. mansont. Rev. Inst. Med. trop. S. Paulo, 26: 51-59, 1984.

4. GENTILINI, M.; DUELO, B.; RICHARD-LENOBLE, D.; BRUCKER, G.; DANIS, M.; NIEL, G. \& MBUNIER, Y. - Assessment of 35.972 RP (Oltipraz), A new antischistosomal drug against Schistosoma haematobium, Schistosoma mansonl and Schistosoms intercalatum. Acta trop. (Basel), 37; 271-274, 1980.

5. KATZ, N.; ROCHA, R. S. \& CHAVES, A. - Doseran. ging clinical trial with oltipraz in schistosomiasis. Rev. Inst. Med. trop. S. Paulo, 24: 40-48, 1982.
6. KATZ, N.; ROCHA, R. S. \& CHAVES, A. - Assessment of oltipraz in schistosomiasts mansoni. Clinical trial Rev. Inst. Med. trop. S. Paulo, 26: 147-151, 1984.

7. REY, J. L.; SELLIN, B.; MOUCHET, F.; SELLIN, E.; SIMONKOVICF, E. \& CHARMOT, G. - Schistosomose urinaire. Premier essai de traitment de masse sur le terrain (Niger). Sem. Hôp. Paris, 59: 707-710, 1983.

8. RHONE-POLENC (LABORATORIES) - Internal report, May, 1981.

Recebido para publicaģåo em 10/7/1985. 